\title{
Optimal Recovery From Microburst Wind Shear
}

Sandeep S. Mulgund

Princeton University

$N 93 \cdot 22574$

Severe low-altitude wind variability represents an infrequent but significant hazard to aircraft taking off or landing. During the period from 1964 to 1985 , microburst wind shear was a contributing factor in at least 26 civil aviation accidents involving nearly 500 fatalities and over 200 injuries. A microburst is a strong localized downdraft that strikes the ground, creating winds that diverge radially from the impact point. The physics of microbursts have only been recently understood in detail, and it has been found that effective recovery from inadvertent encounters may require piloting techniques that are counter-intuitive to flight crews. The goal of this work was to optimize the flight path of a twin-jet transport aircraft encountering a microburst during approach to landing. The objective was to execute an escape maneuver that maintained safe ground clearance and an adequate stall margin during the climb-out portion of the trajectory. 


\section{Trajectory Optimization in Wind Shear}

The objective of trajectory optimization is to determine the state and control histories of a system that minimize a cost function. The choice of the elements of the cost function determines the nature of the optimizing solution. The technique has been used to identify the limits of aircraft performance in wind shear and to determine the control strategies required to achieve such performance. The computation of these optimal trajectories requires global knowledge of the flow field; in other words, the wind components at all points in the aircraft's trajectory must be known in advance. Since this is not possible in practice, the results of trajectory optimization are not immediately useful for realtime control of an aircraft penetrating a wind shear. Nonetheless, the results are valuable for identifying the limits of aircraft performance and for determining the qualitative nature of optimal piloting.

In order to translate the stated goal of recovering from a microburst encounter during final approach into the mathematical expression of a cost function, the objectives of the escape maneuver must be clearly identified. The purpose of the recovery maneuver is to execute a smooth transition from descending to ascending or level flight without stalling the aircraft, saturating the controls, or impacting the ground. Once the aircraft establishes a stable climb, it should maintain an adequate stall margin.

- Determine the state and control histories $\mathbf{x}(t)$ and $\mathbf{u}(t)$ that minimize a cost function:

$$
\left.J=\phi\left[x\left(t_{f}\right)\right]+\int_{t_{0}}^{t_{f}} L[x(t), u(t), t)\right] d t
$$

- Choice of final state penalty $\phi$ and Lagrangian $L$ determine nature of optimizing solution

- Goals of recovery maneuver during approach to landing must be identified to choose a suitable cost function:

- Avoid the ground!

- Perform a stabilized transition from descending to ascending flight

- Maintain an adequate stall margin 


\section{Cost Functions for Aircraft Trajectory Optimization}

The problem of ground avoidance can be solved by maximizing the minimum altitude, which is a maxi-min problem of optimal control. In principle, maximizing the minimum altitude is equivalent to minimizing the peak deviation between some high reference altitude and the aircraft's instantaneous altitude. However, the latter is a mini-max problem of optimal control. The distinction is important when developing an approximate Lagrangian problem of optimal control that can be solved using a Calculus of Variations approach. The optimal solution to the approximate problem shapes the aircraft flight path in such a way that the peak altitude loss of the aircraft is minimized, given the aircraft performance limits and the microburst severity. However, the cost function shown provides little control of the flight path beyond the point of minimum altitude, other than causing the aircraft to climb. Once a positive climb rate is established, it is desirable to regulate airspeed and/or rate of climb. This could have been accomplished by including additional terms in the cost function, but a different approach was taken. A quadratic cost function that considered flight path directly was used. The quadratic cost function had the advantage that it directly weighted the aircraft state variables of interest, and was numerically easier to optimize than a cost function containing larger exponents.

- Ground avoidance $\Rightarrow$ Minimize the peak altitude drop [Miele]:

$$
\min I={ }_{t}^{\max }\left[h_{r e f}-h(t)\right] \quad t_{o} \leq t \leq t_{f}
$$

- Can be reformulated as a Lagrangian problem of optimal control:

$$
J=\int_{t_{0}}^{t_{f}}\left[h_{r e f}-h(t)\right]^{p} d t \quad p>>2 \text { and even }
$$

- Optimal solution shapes aircraft trajectory so that peak difference between $h_{r e f}$ and $h(t)$ is minimized

- Little control over flight path beyond point of minimum altitude

- Motivated use of a Lagrangian that considers flight path directly:

$$
L(x, u)=k_{1}\left[\dot{h}_{r e f}-\dot{h}(t)\right]^{2}+k_{2} q(t)^{2}+k_{3} \delta_{E}(t)^{2}+k_{4} \dot{\delta}_{E}(t)^{2}
$$




\section{Aircraft Equations of Motion}

A three degree-of-freedom model of a twin-jet transport aircraft is used for this study. The aircraft's aerodynamic coefficients are complex nonlinear functions of altitude, Mach number, incidence angles, rotation rates, control deflections, configuration changes (such as gear or flap deflection), and ground proximity. Thrust and elevator dynamics are modeled as first-order lags, and wind shear effects are included in the equations of motion. The wind components and spatial gradients used in the equations of motion are obtained from the Oseguera-Bowles downburst model. This time-invariant analytic model represents an axisymmetric stagnation point flow, based on wind velocity profiles from the Terminal Area Simulation System (TASS). Simulation of microbursts of different size and strength is possible through specification of the radius of the downdraft column $R$, the maximum horizontal wind speed $U_{\max }$, and the altitude of maximum outflow $z_{\max }$.

- Three degree-of-freedom model of a twin-jet transport

Gross weight: $85,000 \mathrm{lb}$

Maximum Takeoff Thrust: $24,000 \mathrm{lb}$

- Aircraft states and controls:

$$
\begin{aligned}
& \boldsymbol{x}=\left[\begin{array}{llllllll}
x & h & u & w & q & \boldsymbol{\theta} & \delta_{E} & \delta_{T}
\end{array}\right] \\
& \boldsymbol{u}=\left[\begin{array}{ll}
\delta_{E} & \delta_{T}
\end{array}\right]_{\text {com }}
\end{aligned}
$$

- Thrust and elevator dynamics modeled as first-order lags

- Wind shear effects included in equations of motion

- Oseguera-Bowles analytical downburst model used to create wind field

- Permits simulation of different microbursts through specification of radius of downdraft column, maximum horizontal wind speed, and height of outflow 


\section{Microburst Encounters During Approach to Landing}

Optimal trajectories were calculated for microburst encounters during approach to landing. The aircraft was initialized on the glide slope outside the core region of the microburst, and it tracked the glide slope until the core was entered. A conjugate gradient algorithm was used to minimize the chosen cost function. This is an iterative technique that requires the existence of a nominal state and control history, which was obtained by performing a recovery using the FAA-recommended maneuver.

- Cost function minimized using a conjugate gradient algorithm

- Nominal state and control histories used to initiate numerical iteration obtained from recoveries performed using FAA technique

- Recovery initiated when aircraft enters core region

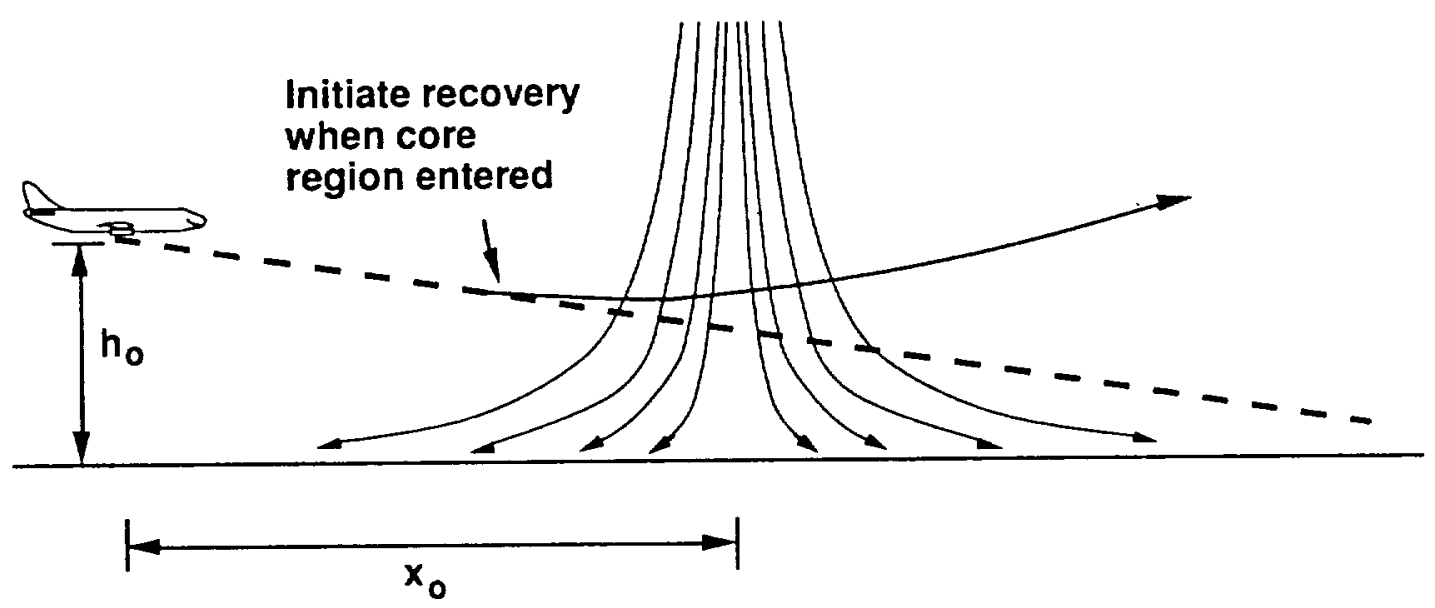




\section{Computation of Optimal Trajectories}

The aircraft was subject to two constraints in the recovery maneuver. First, the elevator deflection was required to lie within a minimum and maximum bound. Second, a minimum airspeed requirement was imposed. The incremental cost function $L_{V}$ was added as a "soft constraint" to prevent excessive violation of the constraint. This function remains zero provided that its argument does not violate a predefined limit. Once the limit is violated, its contribution to the Lagrangian grows quadratically with the magnitude of the violation. The degree to which the aircraft violated the airspeed constraint depended on the relative magnitude of $k_{V}$ and the other weights in the Lagrangian.

- Aircraft subject to two constraints:

$$
\begin{aligned}
& -20^{\circ} \leq \delta_{E} \leq 20^{\circ} \\
& V \geq 125 \text { knots }
\end{aligned}
$$

- Airspeed constraint imposed using a penalty function:

$$
L(\boldsymbol{x}, \boldsymbol{u})=L(\boldsymbol{x}, \boldsymbol{u})+L_{V}(V)
$$

where

$$
L_{V}(V)=\left\{\begin{array}{cc}
0 & V>V_{\min } \\
K_{V}\left[V-V_{\min }\right]^{2} & V \leq V_{\min }
\end{array}\right.
$$

- Contribution of $L v$ to cost grows quadratically with magnitude of constraint violation 


\section{Test Cases}

Optimal trajectories were computed through four microbursts of equal size and varying strength (i.e. varying maximum horizontal wind speed) to illustrate the effect of severity on the shape of the optimal flight paths. The target climb rate during the recovery was set at $5 \mathrm{ft} / \mathrm{sec}$, and the airspeed penalty function threshold was set at 125 knots $(210 \mathrm{ft} / \mathrm{sec})$.

- Illustrate effect of microburst severity on shape of optimal flight paths

- Microburst parameter sets:

$$
\begin{aligned}
R & =3,000 \mathrm{ft} \\
z_{\max } & =150 \mathrm{ft} \\
U_{\max } & =60,70,75, \text { and } 80 \mathrm{ft} / \mathrm{sec}
\end{aligned}
$$

- Aircraft initialized on glide slope just outside core

- Reference rate of climb for escape maneuver set at $5 \mathrm{ft} / \mathrm{sec}$ 


\section{Altitude vs. Time for Optimal Paths through 4 Different Downbursts}

All four optimal flight paths were transitions from descending to ascending flight. However, the introduction of the minimum airspeed penalty function had a significant effect on the aircraft's climb rate during the escape. In the two weakest microbursts, the aircraft was able to maintain the reference climb rate of $5 \mathrm{ft} / \mathrm{sec}(300 \mathrm{ft} / \mathrm{min})$ without violating the penalty function threshold. In the two strongest microbursts, the presence of the airspeed penalty function resulted in a lower climb rate in the escape maneuver.

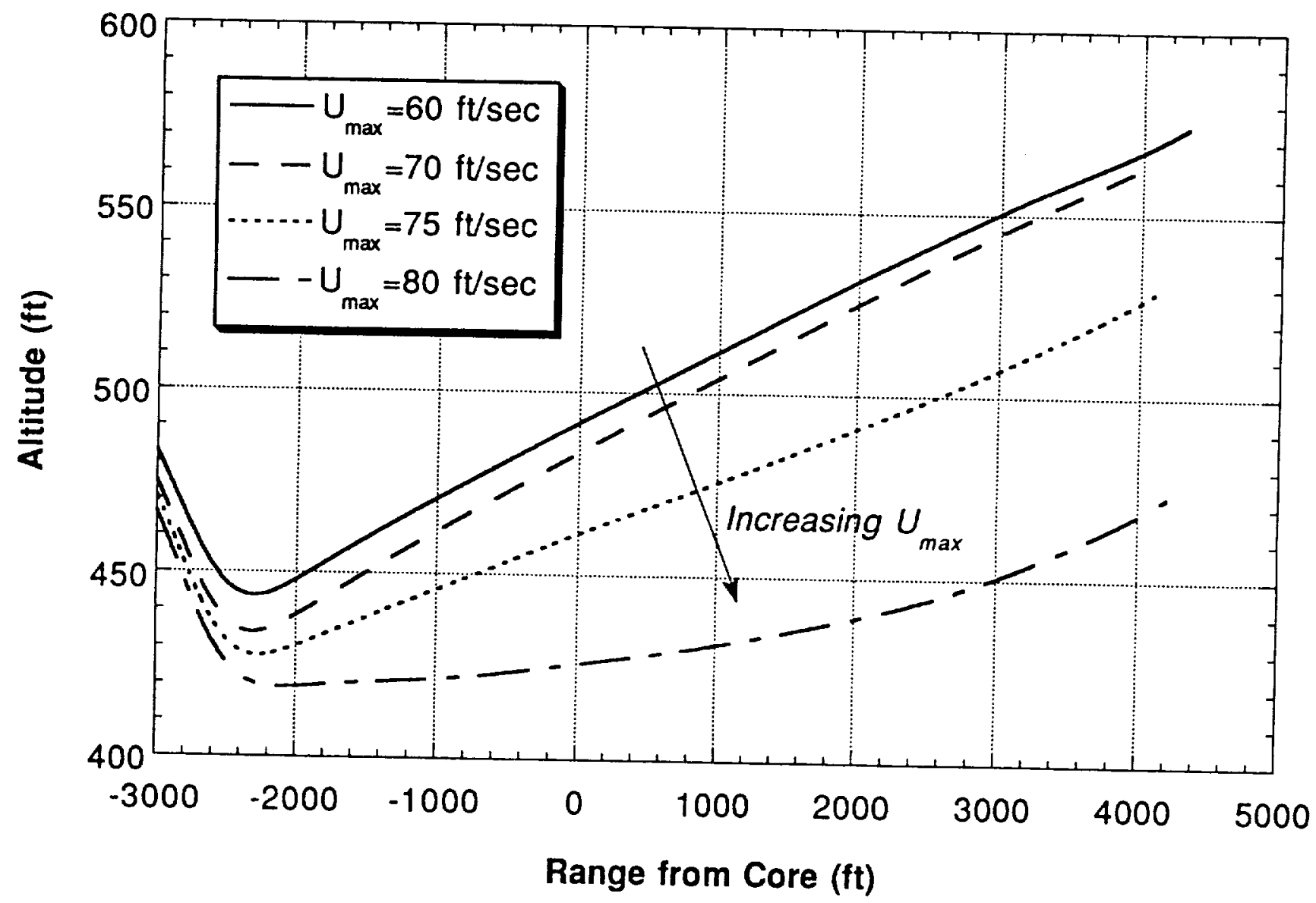




\section{Rate of Climb vs. Time for Optimal Paths through 4 Different Downbursts}

It is apparent here that in the two weaker microbursts, the aircraft was successful in making the transition from descending flight to a $5 \mathrm{ft} / \mathrm{sec}$ ascent. However in the two strongest downbursts, the presence of the airspeed constraint in the cost function caused the optimization algorithm to settle on a target climb rate less than $5 \mathrm{ft} / \mathrm{sec}$.

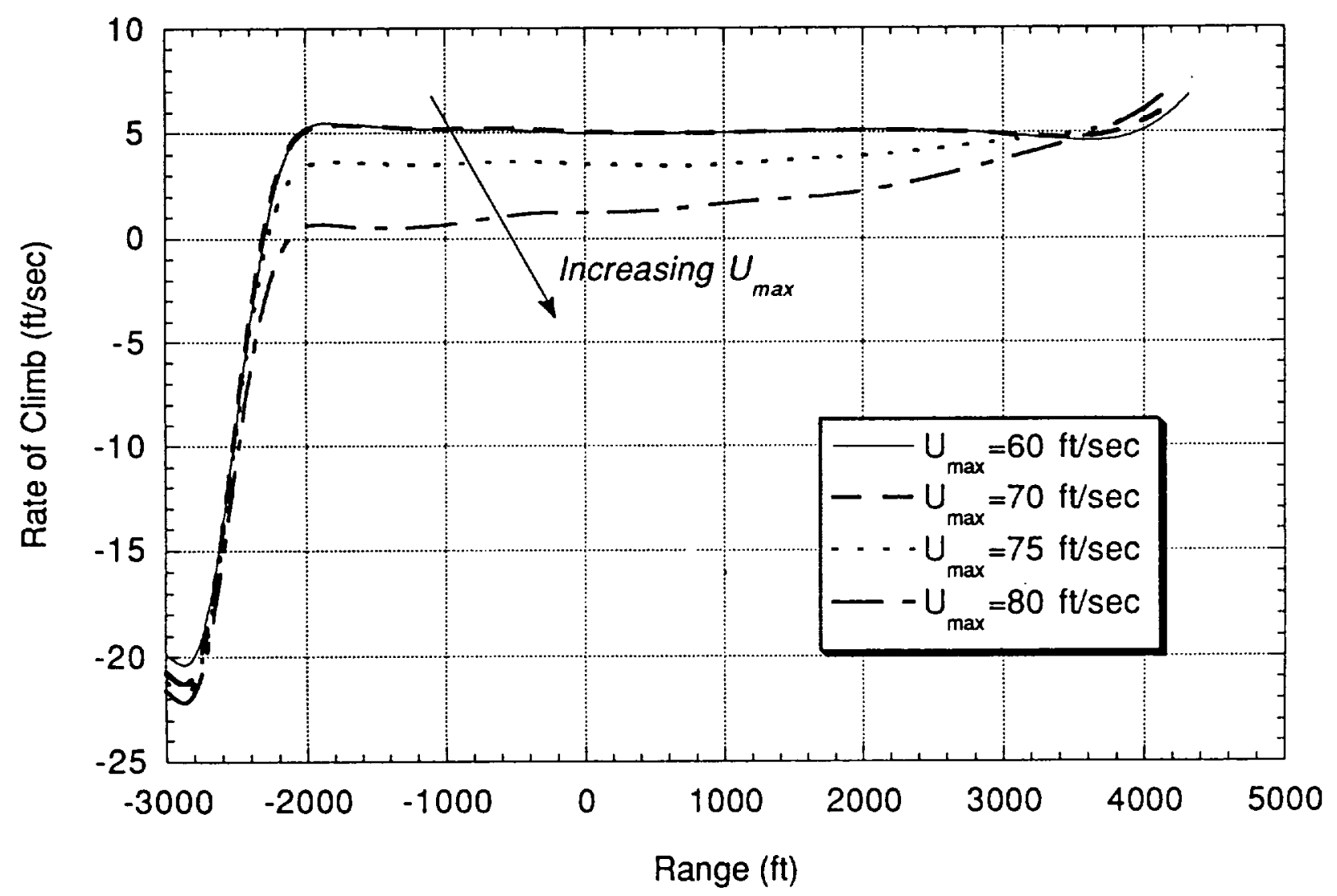




\section{Airspeed vs. Time for Optimal Paths through 4 Different Downbursts}

It can be seen here that in the two weaker microbursts, the escape was successfully accomplished without violating the airspeed penalty function threshold. This was not the case in the two stronger microbursts. In those cases, the airspeed did drop below the threshold somewhat. The degree to which the airspeed dropped below $210 \mathrm{ft} / \mathrm{sec}$ could have been altered by changing the relative magnitude of the climb rate and airspeed weights in the cost function Lagrangian.

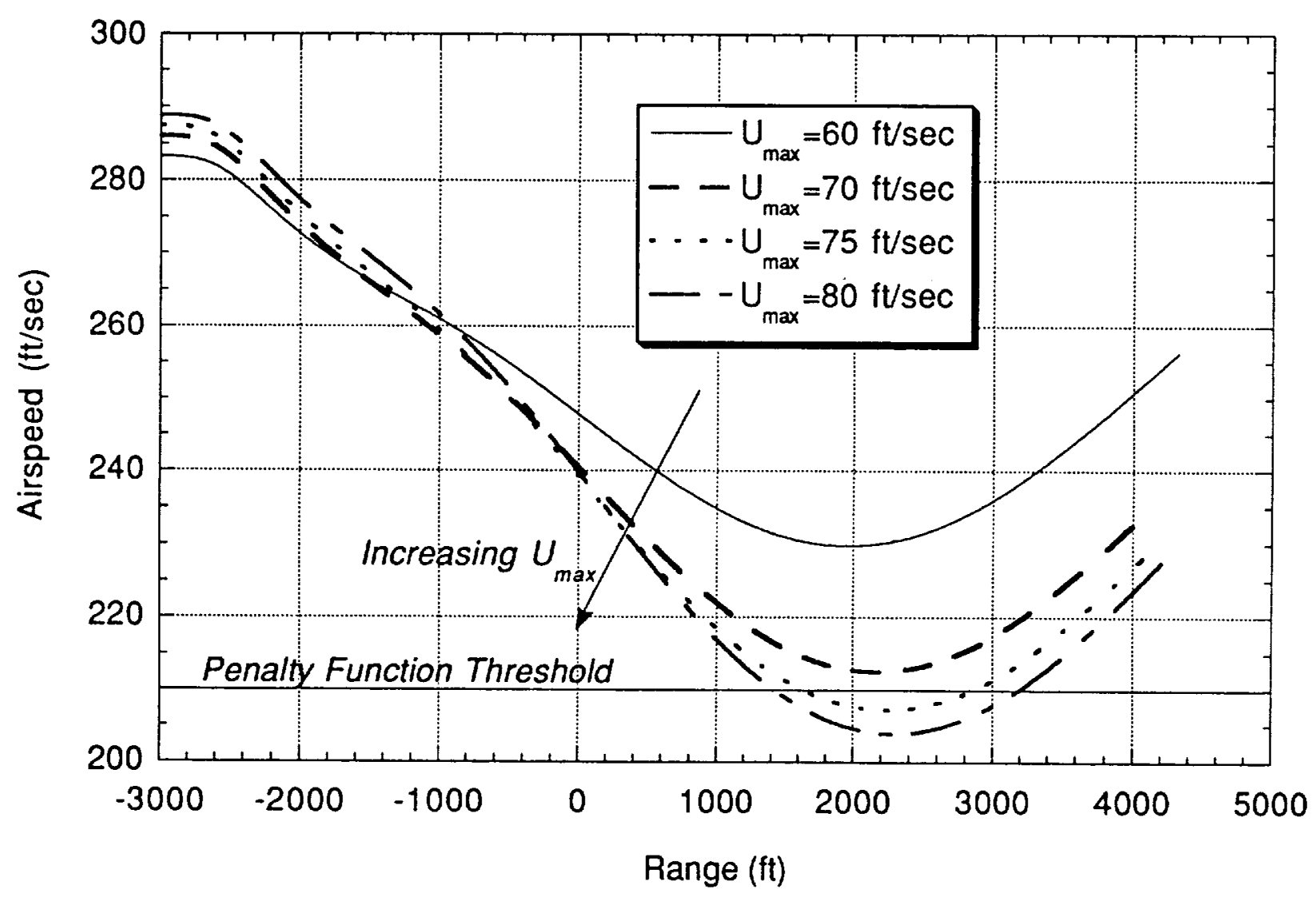




\section{Qualitative Features of the Optimal Flight Paths}

Microburst severity has thus been found to have a significant effect on the nature of an optimal recovery using the given cost function. The optimal maneuver is a rapid transition from descending to level or ascending flight. In weak-to-moderate microbursts, the aircraft easily tracks the reference climb rate throughout the encounter. However, in a severe microburst the aircraft settles on a reduced climb rate through much of the encounter to prevent excessive airspeed loss. While these results are useful for providing insight into the nature of optimal recovery techniques, they are not immediately useful for real-time feedback control. Global knowledge of the flow field is required for optimization. Even with the advent of forward-look sensors, such detailed information about a microburst's structure will not be available. Furthermore, optimization is itself an iterative time-consuming process.

- Rapid transition from descending to level or ascending flight

- Targeted rate of climb during escape depends on wind shear severity

Weak to moderate $\Rightarrow$ Aircraft reaches $5 \mathrm{ft} / \mathrm{sec}$ climb rate

Severe to very severe $\Rightarrow$ Aircraft reaches a lower climb rate

- Lower climb rate in severe microbursts results in reduced violation of minimum airspeed constraint

\section{OK, but...}

- Global knowledge of flow field required for optimization

- Results not immediately applicable to real-time feedback control 


\section{Future Work: Neural Networks for Real-Time Flight Guidance}

Although the results of trajectory optimization are not immediately applicable to feedback control, it should be possible to make use of the insights gained through the optimization study somehow. One idea under consideration is to train an artificial neural network with the results of trajectory optimization. The inputs to the network would be aircraft state and wind information, and the outputs might be throttle and flight path commands. It should be possible to parametrize microbursts according to size and severity, and then have the neural network generate flight path commands according to the aircraft position within the flow field. Forward-look information on wind intensity might assist in the flight path planning.

- Train neural network with results of trajectory optimization

- Can parametrize microbursts according to size and severity

- Network generates flight path angle commands according to position within flow field

- Availability of forward-look information could assist in flight-path planning

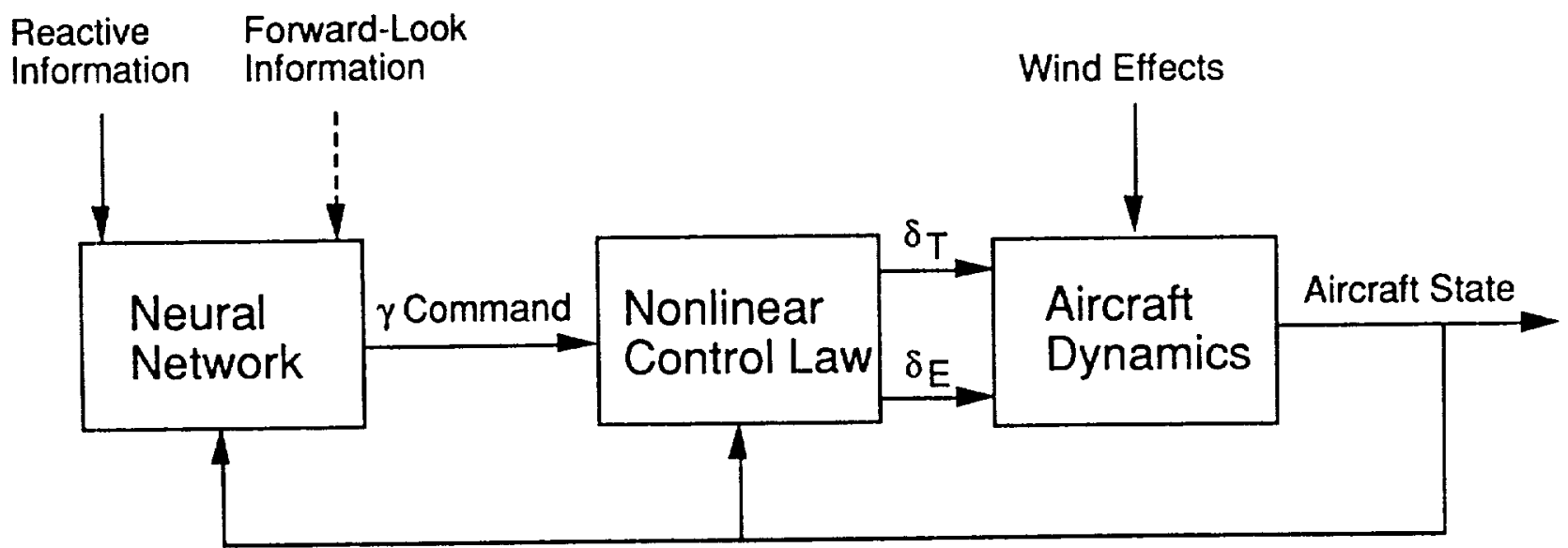

